\title{
Saúde bucal de pacientes oncológicos atendidos no Hospital de Câncer Infantojuvenil de Barretos
}

\section{Oral health of oncology patients attended at the Barretos Cancer Hospital of Children and youth}

\begin{abstract}
Natália Shizu Kusuke ${ }^{1}$ - Autor Principal (nskusuke@outlook.com) telefone: (11) 991870897, William Eduardo Pirola ${ }^{1}$, Thaisa Theodoro De Oliveira, ${ }^{1}$ Fernanda Oliveira Lemos ${ }^{2}$ Hélio Massaiochi Tanimoto ${ }^{3}$ Miriam Eiko Katuki Tanimoto ${ }^{4}$ Karina Silva Moreira Macari ${ }^{5}$

1- Residente do Hospital de Câncer de Barretos. R. Antenor Duarte Viléla, 1331 - Dr. Paulo Prata, Barretos - SP, 14784-400

2- Membro da equipe de Odontologia do Hospital de Câncer de Barretos

3- Chefe do departamento de Odontologia do Hospital de Câncer de Barretos.

4- Professora da Faculdade de odontologia de Barretos - UNIFEB Av. Prof. Roberto Frade Monte, 389 - Aeroporto, Barretos - SP, 14783-226

5- Orientadora e Dentista responsável pelo departamento de Odontopediatria do Hospital de Câncer Infantojuvenil de Barretos
\end{abstract}

\section{Resumo}

Objetivo: Avaliar a condição bucal dos pacientes que frequentaram o Hospital de Câncer Infantojuvenil, de Barretos do período de novembro de 2013 a julho de 2014. Métodos: A Coleta foi realizada através do Índice de biofilme, gengival e Índice de Cárie (CPO-D e ceo-d). Resultados: Dos 104 pacientes, $31,7 \%(n=33)$ possuíam diagnóstico benigno, enquanto $68,3 \%(n=71)$, diagnóstico maligno. O índice de biofilme obteve média geral de $0,94(\mathrm{DP}=0,40)$. Nenhum dos pacientes apresentou faces de dentes livres de biofilme. A maioria possuía gengivas com moderada inflamação, correspondendo a 59,6\% dos casos. Com relação aos índices de cárie, o índice de ceo-d teve uma média geral de 1,60 (DP=2,15) e o CPO-D com média geral de 4,06 (DP=3,68), neste estudo. Conclusão: Através do estudo, pode-se observar que a saúde bucal da população que frequenta o Hospital Infantojuvenil de Barretos não corresponde ao ideal, apresentando índice de biofilme, índice gengival e índice de cárie consideráveis, o que aumenta o risco de complicações durante o tratamento oncológico.

Palavras-chave: Odontologia pediátrica, saúde bucal, Índice Periodontal, cárie dentária.

\begin{abstract}
Objective: To evaluate the oral health of patients seen at the Children's Cancer Hospital of Barretos in the period between November, 2013 and July, 2014. Methods: Collection was performed with the biofilm, gingival, and caries indexes (DMFT and dmft). Results: Of 104 patients, $31.7 \%(\mathrm{n}=33)$ had benign diagnosis, while $68.3 \%(\mathrm{n}=71)$ had malignant diagnosis. The oral biofilm index presented an overall average of $0.94(\mathrm{SD}=0.40)$. None of the patients presented teeth surfaces free of plaque. Most gums presented moderate inflammation, which accounts for $59.6 \%$ of cases. Regarding decay rates, this study found an overall average of $1.60(\mathrm{SD}=2.15)$ for the $\mathrm{dmft}$ index and of $4.06(\mathrm{SD}=3.68)$ for the DMFT. Conclusion: The study showed that the oral health of the population in the Children's Hospital of Barretos does not correspond to the ideal, having presented significant biofilm, gingival, and caries indexes and considerable decay rate, which increases the risk of complications during cancer treatment.
\end{abstract}

Keywords: Pediatric dentistry, oral health, periodontal index, dental caries.

Autor para correspondência: Natália Shizu Kusuke e-mail: nskusuke@outlook.com

Recebido em: 14/09/2015

Aceito para publicação em: 01/03/2016

https://doi.org/10.4322/1980-0029.172015 


\section{Introdução}

O câncer infantojuvenil é a primeira causa de morte por doença entre crianças e adolescentes de 1 a 19 anos, para todos os países considerados desenvolvidos e em desenvolvimento, assim como no Brasil (Instituto Nacional de Câncer, 2013). A mortalidade e a morbidade se relacionam com o tratamento e a condição de saúde sistêmica e bucal (Almeida et al., 2014). A cavidade bucal, por ser um nicho de proliferação bacteriana, pode desencadear problemas bucais decorrentes do tratamento, sejam eles sistêmicos ou locais.

Cerca de $40 \%$ dos pacientes que fazem quimioterapia desenvolvem efeitos colaterais bucais. Este número eleva-se para mais de $90 \%$ quando a criança se encontra abaixo dos 12 anos de idade (Sonis et al., 1996). Alguns estudos apontam que, se houver protocolo de atendimento odontológico antes e durante a terapêutica empregada, é possível reduzir os riscos de complicações bucais e a severidade da mucosite (Levy-Polack et al., 1998; Frazão et al., 2012).

O estudo de Lopes et al. (2012) avaliou as manifestações orais decorrentes do

tratamento de câncer em pacientes de 6 a 12 anos de idade. Dos 24 indivíduos avaliados, 15 apresentaram uma condição oral desfavorável e tiveram manifestações orais decorrentes da quimioterapia, enquanto dos seis que apresentaram condição favorável, apenas três relataram a presença delas, mostrando redução de $50 \%$ das manifestações.

A grande variedade de microrganismos presentes na cavidade bucal faz com que esta seja um portal de entrada significante para agentes infecciosos durante o período de mielossupressão, podendo causar infecções virais, bacterianas e fúngicas. A quantidade de biofilme acumulada por três dias favorece o aparecimento de gengivite e a doença periodontal ativa, somada à baixa imunidade (Kroetz \& Czlusniak, 2003).

Os cuidados odontológicos devem ser direcionados para promover e manter a integridade do tecido e prevenir complicações bucais, melhorando a qualidade de vida (Lobão et al., 2008). Portanto, avaliar a condição bucal de pacientes atendidos no Hospital de Câncer Infantojuvenil de Barretos Presidente Luiz Inácio Lula da Silva é de suma importância para a definição de um protocolo.

\section{Material e método}

O estudo foi realizado de maneira descritiva e a coleta de maneira prospectiva de amostragem por conveniência, com o intuito de descrever a qualidade bucal dos pacientes que passaram por atendimento no Hospital de Câncer Infantojuvenil de Barretos Presidente Luiz Inácio Lula da Silva, no período de novembro de 2013 a julho de 2014.

Para o cálculo amostral, foi utilizado o número de pacientes novos atendidos por mês no Hospital - em média, 15 pacientes novos ao mês - e, considerando a margem de idade definida, foi calculado, em média, o número de 100 pacientes com o estudo.

O estudo foi aprovado pelo Comitê de Ética em Pesquisa (CEP) do Hospital de Câncer de Barretos sob o protocolo 739/2013. Não há conflito de interesses no presente artigo.

Os participantes foram selecionados de acordo com os critérios de elegibilidade: crianças com, no mínimo, cinco anos e que já possuíam o primeiro molar permanente irrompido; indivíduos com até 25 anos de idade podiam participar, desde que estivessem no hospital para tratamento ou diagnóstico da doença, e que não houvessem recebido orientação do Departamento de Odontologia. Após a aplicação do Termo de Consentimento Livre e Esclarecido (TCLE), os participantes foram encaminhados para o Departamento de Odontologia a fim de realizar a coleta de dados como uma primeira consulta, sendo uma única pesquisadora responsável, totalizando seu valor Kappa em 80\%.

Foram coletadas questões socioeconômicas, demográficas e relacionadas à saúde bucal e sistêmica do paciente, a partir de um formulário. A coleta clínica foi realizada através do índice de biofilme e índice gengival, aplicados de acordo com o Índice de Silness \& Löe (1964) e (Pereira, 2009).

Quatro dentes índices foram avaliados, correspondendo às dentições mista e permanente. Os pontos analisados em cada dente foram: vestibular, distal, mesial e palatino/lingual. Só foram analisados dentes com o terço gengival da coroa íntegra. Para cada ponto de avaliação, foi anotado um valor. $\mathrm{O}$ valor do índice foi calculado pela somatória dos valores individuais divididos por quatro e depois divididos por seis, sendo o total dos dentes avaliados.

O Índice de Cárie (ceo-d e CPO-D) foi realizado de acordo com o preconizado pela World Health 
Organization (1997). Uma profilaxia prévia foi realizada para maior fidelidade do exame. Foram avaliados todos os dentes e espaços vazios, iniciando no último dente irrompido, exceto os terceiros molares, de acordo com a faixa etária do indivíduo. Os valores foram somados tendo como resultado a história da doença cárie individual. Todos os índices foram coletados com o auxílio de espelho clínico e sonda exploradora de ponta romba.

Os dados foram analisados considerando suas medidas descritivas. Foram calculados a média, o desvio padrão, o mínimo, o máximo e os quartis para as variáveis quantitativas. Já para as variáveis qualitativas, foram realizadas tabelas de frequência, através do Software SPSS 21.0.

\section{Resultados}

Foram coletados dados de 104 participantes e, dentre eles, $49 \%(n=51)$ eram do sexo feminino e $51 \%(n=53)$, do sexo masculino. O intervalo entre as idades variou de 5 a 25 anos, sendo a média de idade de 14 anos. A prevalência foi de leucodermos $45,6 \%(n=47)$ e faiodermos $39,8 \%$ $(\mathrm{n}=41)$. A maioria dos participantes tinha como procedência a região Sudeste do Brasil, $55,8 \%$ $(n=58)$, e a região Norte, $23,1 \%(n=24)$.

Com relação aos responsáveis pelos participantes menores de 18 anos, a grande maioria era casada, $69,5 \%(n=66), 30,4 \%$ trabalhavam com prestação de serviços $(n=28)$ e $22,8 \%$ consideravam-se como donas de casa $(\mathrm{n}=21)$. A renda familiar concentrou-se em torno de 1 a 2 salários mínimos, correspondendo a $63,3 \%(n=69)$ dos casos. Dentre os demais, $11,5 \%$ $(\mathrm{n}=12)$ não quiseram informar; $12,5 \%(\mathrm{n}=13)$ recebiam de 3 a 5 salários mínimos; 5,8\% $(\mathrm{n}=6)$ recebiam mais de seis salários mínimos, e 3,8\% $(n=4)$ recebiam menos de um salário.

Com relação às informações clínicas, 31,7\% $(n=33)$ dos participantes apresentaram diagnóstico benigno, enquanto $68,3 \%(\mathrm{n}=71)$, diagnóstico maligno da doença. A maioria pertenceu ao grupo de tumores sólidos, $63,4 \%(\mathrm{n}=45)$, sendo os tumores líquidos, de $36,6 \%(n=26)$. Os tumores hematológicos corresponderam a $36,6 \%(n=26)$, seguidos por tumores do sistema nervoso central, $16,9 \%(n=12)$, e ósseos, $14,1 \%(n=10)$, ocupando a terceira posição das amostras.

A maioria disse escovar os dentes diariamente, duas vezes ao dia, com 51,5\% $(\mathrm{n}=50)$. Grande parte não usava o fio dental, 76,9\% $(\mathrm{n}=80)$. Dentre as crianças até 12 anos, $11,9 \%(n=5)$, relataram receber sempre o auxílio dos pais (Tabela 1).

Com relação à saúde bucal, o índice de biofilme bucal correspondeu a uma média geral de $0,94(d p=0,40)$. Em sua maior parte, foi caracterizado com pouco biofilme no momento da avaliação, com $68,3 \%$ dos participantes ( $n=71$ ), sendo visível apenas com o uso de sonda; $29,8 \%$ $(\mathrm{n}=31)$ possuíam moderado acúmulo de biofilme, e o nível de biofilme severo foi observado em $1,9 \%(n=2)$ (Tabela 2).

A saúde gengival correspondeu, em sua média geral, por 1,28 (desvio padrão $=0,67)$, sendo os

TABELA 1 - Número e porcentagem de variáveis relacionadas a hábitos de higiene bucal dos indivíduos (Barretos-SP, novembro de 2013 a julho de 2014)

\begin{tabular}{lccc}
\hline \multicolumn{1}{c}{ Variável } & Categoria & (N) & \% \\
\hline Escovação diária & Sim & 97 & 93,3 \\
& Não & 7 & 6,7 \\
No de vezes ao dia & Uma Vez & 18 & 18,6 \\
& Duas Vezes & 50 & 51,5 \\
& Três Vezes & 28 & 28,9 \\
Escovação da língua & Quatro Vezes & 1 & 1 \\
& Sim & 24 & 80,8 \\
Uso do fio dental & Não & 80 & 19,2 \\
& Sim & 24 & 23,1 \\
Uso de solução bucal & Não & 80 & 76,9 \\
& Sim & 17 & 16,3 \\
Auxílios dos responsáveis & Não & 87 & 83,7 \\
& Nunca & 21 & 50,0 \\
& Às vezes & 16 & 38,1 \\
& Sempre & 5 & 11,9 \\
\hline
\end{tabular}

*Pacientes acima de 12 anos foram excluídos. 
TABELA 2 - Número, média, desvio padrão (DP) e porcentagem com relação aos índices de biofilme e gengival, dos indivíduos (Barretos-SP, novembro de 2013 a julho de 2014)

\begin{tabular}{lclcr}
\hline \multicolumn{1}{c}{ Variável } & Média (DP) & \multicolumn{1}{c}{ Categoria } & (N) & \% \\
\hline Índice de Biofilme & $0,94(0,40)$ & Pouco & 71 & 68,3 \\
& & Moderado & 31 & 29,8 \\
& & Severo & 2 & 1,9 \\
Índice gengival & \multirow{2}{*}{$1,28(0,67)$} & Sem inflamação & 3 & 2,9 \\
& & Leve inflamação & 32 & 30,8 \\
& & Inflamação moderada & 62 & 59,6 \\
\hline
\end{tabular}

TABELA 3 - Número, média, desvio padrão (DP) e porcentagem de dentes cariados, perdidos, restaurados e CPO-D (Barretos-SP, novembro de 2013 a julho de 2014)

\begin{tabular}{|c|c|c|c|c|}
\hline Variável & Média (DP) & Categoria & $(\mathrm{N})$ & $\%$ \\
\hline \multirow{5}{*}{$\begin{array}{l}\text { Permanentes } \\
\text { cariados }\end{array}$} & $1,91(2,26)$ & $=0$ & 39 & 37,57 \\
\hline & & $\geq 1 \mathrm{e}<3$ & 31 & 31,7 \\
\hline & & $\geq 3$ e $<5$ & 19 & 18,3 \\
\hline & & $\geq 5$ e $<7$ & 9 & 8,7 \\
\hline & & $\geq 7$ & 4 & 3,8 \\
\hline \multirow{5}{*}{$\begin{array}{l}\text { Permanentes } \\
\text { restaurados }\end{array}$} & $1,98(2,94)$ & $=0$ & 46 & 44,2 \\
\hline & & $\geq 1 \mathrm{e}<3$ & 29 & 27,9 \\
\hline & & $\geq 3 \mathrm{e}<5$ & 13 & 12,5 \\
\hline & & $\geq 5 \mathrm{e}<7$ & 8 & 7,7 \\
\hline & & $\geq 7$ & 8 & 7,7 \\
\hline \multirow{3}{*}{$\begin{array}{l}\text { Permanentes } \\
\text { perdidos }\end{array}$} & $0,17(0,56)$ & $=0$ & 93 & 89,4 \\
\hline & & $\geq 1 \mathrm{e}<3$ & 9 & 8,7 \\
\hline & & $\geq 3$ & 2 & 1,9 \\
\hline \multirow[t]{4}{*}{ CPO-D } & $4,06(3,68)$ & $=0$ & 19 & 18,3 \\
\hline & & $\geq 1 \mathrm{e}<3$ & 27 & 26 \\
\hline & & $\geq 3 \mathrm{e}<6$ & 24 & 23,1 \\
\hline & & $\geq 6$ & 34 & 32,7 \\
\hline
\end{tabular}

TABELA 4 - Número, média, desvio padrão e porcentagem de dentes cariados, restaurados, perdidos e ceo-d dos indivíduos (Barretos-SP, novembro de 2013 a julho de 2014)

\begin{tabular}{lcccc}
\hline \multicolumn{1}{c}{ Variável } & Média (DP) & Categoria & (N) & \% \\
\hline Decíduos cariados & $1,02(1,65)$ & $=0$ & 26 & 57,8 \\
& & $>0 \mathrm{e}<3$ & 11 & 24,4 \\
Decíduos restaurados & $0,42(0,86)$ & $\geq 3$ & 8 & 17,8 \\
& & $\geq 1$ & 46 & 44,2 \\
Decíduos perdidos & $0,20(0,94)$ & $=0$ & 29 & 27,9 \\
& & $\geq 1$ & 42 & 93,3 \\
ceo-d & $1,60(2,15)$ & $=0$ & 3 & 6,7 \\
& & $\geq 1 \mathrm{e}<3$ & 21 & 46,7 \\
& & $\geq 3$ & 13 & 28,9 \\
\hline
\end{tabular}

casos sem presença de inflamação correspondendo a 2,9\% $(n=3)$. Gengivas com eritemas e leve inflamação corresponderam a 30,8\% dos casos $(n=32)$, enquanto gengivite com sangramento ao passar a sonda correspondeu a 59,6\% $(n=62)$ dos casos (Tabela 2).
Com relação aos índices de cárie, o CPO-D teve uma média geral de 4,06 (dp=3,68) e o ceo-d, com média geral de 1,60 $(\mathrm{dp}=2,15)$ (Tabelas 3 e 4).

Foi relacionada a questão socioeconômica com a presença de gengivite e a história de cárie (Tabelas 5, 6 e 7). Observa-se que a grande maioria 
Tabela 5 - Correlação entre renda familiar e Índice gengival

\begin{tabular}{|c|c|c|c|c|c|c|}
\hline & Renda familiar & & Índice & ival & & Totol \\
\hline & (Salários mínimos) & 0 a 0,9 & 1 a 1,99 & 2 a 2,99 & 3 & lotal \\
\hline & Menos de um salário & 1 & 2 & 1 & 0 & 4 \\
\hline & 1 a 2 & 18 & 37 & 12 & 2 & 69 \\
\hline & 3 a 5 & 6 & 5 & 2 & 0 & 13 \\
\hline & Mais de 6 & 2 & 3 & 1 & 0 & 6 \\
\hline & Não quis informar & 3 & 7 & 2 & 0 & 12 \\
\hline Total & & 30 & 54 & 18 & 2 & 104 \\
\hline
\end{tabular}

Tabela 6 - Correlação entre renda familiar e CPO-D

\begin{tabular}{|c|c|c|c|c|c|}
\hline \multirow{2}{*}{$\begin{array}{c}\text { Renda familiar } \\
\text { (salários mínimos) }\end{array}$} & \multicolumn{4}{|c|}{ CPO-D } & \multirow{2}{*}{ Total } \\
\hline & $=\mathbf{0}$ & $\geq 1 \mathrm{e}<3$ & $\geq 3 \mathrm{e}<6$ & $\geq 6$ & \\
\hline Menos de um salário & 2 & 0 & 0 & 2 & 4 \\
\hline 1 a 2 & 13 & 19 & 14 & 23 & 69 \\
\hline 3 a 5 & 3 & 3 & 4 & 3 & 13 \\
\hline Mais de 6 & 0 & 1 & 4 & 1 & 6 \\
\hline Não quis informar & 1 & 4 & 2 & 5 & 12 \\
\hline Total & 19 & 27 & 24 & 34 & 104 \\
\hline
\end{tabular}

Tabela 7 - Correlação entre renda familiar e ceo-d

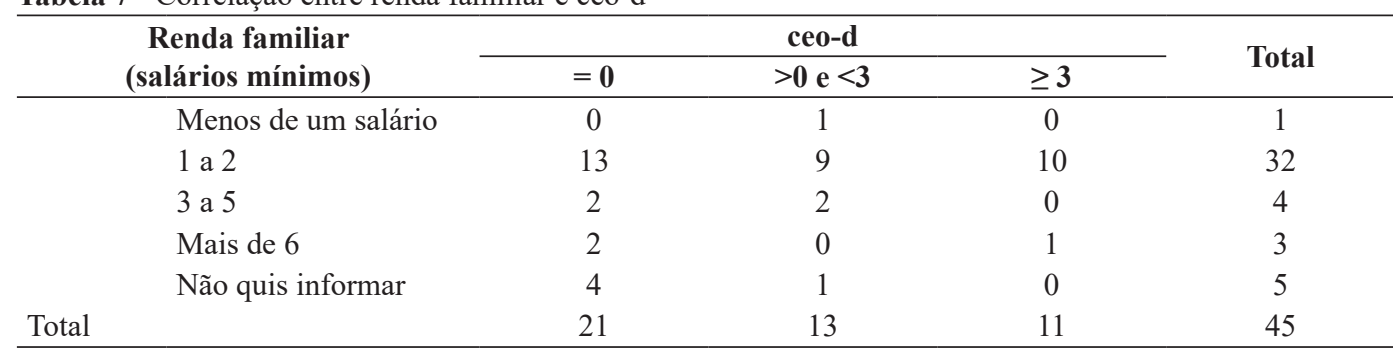

da população, entre um e dois salários mínimos, apresenta início de gengivite com sangramento à sondagem (de 1 a 1,99). Similarmente, há a presença de seis ou mais dentes permanentes com história de cárie, na população de mesma renda, sendo que a mesma história se repete na dentição decídua, predominando três ou mais cáries em dentes decíduos, em 10 dos 45 indivíduos com dentes decíduos na cavidade bucal.

\section{Discussão}

A literatura mostra que os tumores mais incidentes em crianças primeiramente pertencem ao grupo líquido, no qual estão a leucemia e o linfoma; depois, ao grupo dos sólidos, no qual se encontram os demais cânceres, como os de Sistema Nervoso Central, que ocupam o terceiro lugar no grupo de tumores mais incidentes no Brasil (Instituto Nacional de Câncer, 2013). Este estudo seguiu a mesma incidência, com a grande maioria pertencendo ao grupo dos tumores hematológicos, e tumores do SNC e ósseos ocupando a terceira posição.

No estudo de Pels \& Mielnik-Blaszczak (2012), encontrou-se pior higiene bucal em crianças com leucemia que residiam em áreas rurais, comparadas às urbanas. No presente estudo, estas diferenças não ficaram muito claras, uma vez que não se dividiu a origem dos participantes por área urbana e rural, porém, com relação à questão socioeconômica, a população com menor renda foi caracterizada por pior qualidade de saúde bucal.

Rocha (2014), em seu estudo, avaliou a saúde bucal de pacientes em quimioterapia. Na avaliação da condição de saúde gengival, no tempo inicial, o índice gengival médio foi de 0,74 , valor mais baixo se comparado a este estudo $(1,28)$, o qual demonstra uma grande falha na higiene bucal da população investigada. A higiene bucal adequada 
é imprescindível nessas ocasiões de tratamento oncológico, principalmente pela imunossupressão.

Nos resultados encontrados por Ximenes et al. (2008) e Rodrigues et al. (2011), observou-se que cerca de $92 \%$ dos acompanhantes das crianças não receberam qualquer informação sobre saúde bucal durante a internação, portanto, um contato primário com o dentista antes do início do tratamento oncológico é de suma importância para orientar o acompanhante e a criança, além de realizar a prevenção de infecções durante o tratamento

Em um estudo realizado por Lobão et al. (2008), verificou-se a saúde bucal de crianças com leucemia e realizou-se o acompanhamento. Os índices CPO-D e ceo-d médio foram de 2,5 e 2,2, respectivamente, e a atividade de cárie presente no início da avaliação foi de $67,7 \%$. O resultado foi semelhante ao do presente estudo, sendo a porcentagem de cárie ativa e com necessidade de tratamento de $62,5 \%(n=63)$ em dentes permanentes e $42,2 \%$ (n=19) em dentição decídua, demonstrando que mais da metade da população investigada possuía necessidade de tratamento odontológico, previamente ao oncológico.

No estudo de Figueiredo \& Nogueira (2013), em que se comparava a qualidade bucal de crianças em tratamento oncológico, antes e depois do tratamento, o índice gengival no exame inicial foi de 0,492 e, no final, 0,549. Com relação ao índice de cárie, o CPO-D médio no exame inicial foi de 2,166 e, no segundo, aumentou para 2,625. No ceo-d médio, o exame inicial foi de 2,814 e, no segundo, de 3,25; portanto, houve um breve aumento desde a primeira avaliação para a segunda, constatando que pode ocorrer piora da qualidade bucal durante o tratamento oncológico. Por isso, o acompanhamento com a equipe de Odontologia deve ser seguido nestes pacientes (Rodrigues et al., 2011).

A OMS estabelecia o CPO-D $=3,0$, aos 12 anos de idade, situada entre prevalência moderada como meta a ser atingida, até o ano de 2000 (Ministério da saúde, 1997 e 2003). No ano de 2003, o estudo do SB BRASIL mostrou que a meta foi atingida para crianças de cinco e 12 anos, correspondendo à média de 2,8 de dentes cariados (Brasil, 2003).

Outro estudo da população quanto à saúde bucal foi realizado pelo SB BRASIL (2010) e verificou-se que, aos cinco anos de idade, o ceo-d médio era de 2,43. Crianças brasileiras de 12 anos de idade e adolescentes de 15 a 19 anos apresentavam, respectivamente, em média, 2,07 e 4,25 dentes com experiência de cárie dentária no ano de 2010. A dentição decídua livre da experiência cárie correspondia a 46,6\% e, aos 12 anos, $43,5 \%$ das crianças apresentavam a dentição permanente livre de cárie.

Neste estudo, a experiência de cárie foi ausente em 18,3\% ( $\mathrm{n}=19)$ dos dentes permanentes e $46,7 \%(n=21)$ dos dentes decíduos, mostrando uma piora com relação aos dentes permanentes e pouca mudança em dentes decíduos, com relação ao SB BRASIL 2010. Já existem campanhas que remetem a programas de prevenção, tratamento especializado e reabilitação dessas crianças, para manutenção desses índices (Brasil, 1997, 2003, 2010). Contudo, neste estudo, $10,6 \%(n=11)$ das crianças já possuíam algum dente permanente perdido por atividade de cárie, enquanto que, nos dentes decíduos, $6,7 \%(\mathrm{n}=3)$ das crianças haviam perdido algum dente.

Apesar de não terem sido observados índices altos entre crianças e jovens investigados, a presença de lesões cariosas eleva os níveis de infecções que podem acometê-los durante o tratamento. Além disso, é propício o aumento dessas lesões, requerendo o tratamento e a prevenção de doenças orais como qualidade de vida para o paciente durante o tratamento oncológico.

\section{Conclusão}

Através do estudo, pode-se observar que a saúde bucal da população que frequenta o Hospital Infantojuvenil de Barretos não corresponde ao ideal, apresentando índice de biofilme, índice gengival e índice de cárie consideráveis, o que aumenta o risco de complicações durante o tratamento.

\section{Referências}

Almeida, T. F., Torres, A. S., Silva, R. A., Wanderley, F. G. C., \& Fonseca, E. M. (2014). Avaliação dos cuidados de saúde bucal em pacientes pediátricos hospitalizados. Revista de Ciências Médicas e Biológicas, 13(1), 72-77.

Brasil. Ministério da Saúde. Rede Interagencial de Informações para a Saúde. (1997). Indicadores e dados básicos. Qualificação de Indicadores do IDB-1997. Brasília: Ministério da Saúde. Retrieved in 2015, February 11, from http://tabnet.datasus. gov.br/cgi/idb1997/morb/fqd10.htm 
Brasil. Ministério da Saúde. Secretaria de Atenção à Saúde. Departamento de Atenção Básica. (2003). Projeto SB Brasil 2003: condições de saúde bucal da população brasileira - Resultados principais. Brasília: Ministério da Saúde; 2003.

Brasil. Ministério da Saúde. Secretaria de Atenção à Saúde. Departamento de Atenção Básica. (2010). Projeto SB Brasil 2010: condições de saúde bucal da população brasileira - Resultados principais. Brasília: Ministério da Saúde; 2010.

Figueiredo, P. B. A., \& Nogueira, A. (2013). Prevalência de neoplasias, cárie e gengivite em pacientes oncológicos pediátricos. Pesquisa Brasileira em Odontopediatria e Clínica Integrada, 13(2), 141-146.

Frazão, C. O. B., Alfaya, T. A., Costa, R. C., Rocha, M. L., Gouvêa, C. V. D., \& Morais, A. P. (2012). Pacientes oncológicos pediátricos: manifestações bucais da terapia antineoplásica. Revista Saúde e Pesquisa., 5(3), 587-592.

Instituto Nacional de Câncer - INCA. (2013). Câncer infantil. Retrieved in 2013, April 10, from http://www2.inca.gov.br/wps/wcm/connect/ tiposdecancer/site/home/infantil

Kroetz, F. M., \& Czlusniak, G. D. (2003). Alterações bucais e condutas terapêuticas em pacientes infanto-juvenis submetidos a tratamentos anti-neoplásicos. Publicatio UEPG. Ciências Biológicas e da Saúde, 9(2), 41-48.

Levy-Polack, M. P., Sebelli, P., \& Polack, N. (1998). Incidence of oral complications and application of a preventive protocol in children with acute leukemia. Special Care in Dentistry, 5(18), 189-193.

Lobão, D. S., Oliveira, B. M., Massara, M. L. A., Viana, M., \& Nunes, L. (2008). Condições da cavidade bucal e acompanhamento odontológico de crianças com leucemia linfocística aguda. Revista Médica de Minas Gerais, 18, S25-S32.

Lopes, I. A., Nogueira, D. N., \& Lopes, I. A. (2012). Manifestações orais decorrentes da quimioterapia em crianças de um Centro de Tratamento Oncológico. Pesquisa Brasileira em Odontopediatria e Clinica Integrada, 12(1), 13-19.

Pels, E., \& Mielnik-Blaszczak, M. (2012). Oral hygiene in children suffering from acute lymphoblastic leukemia living in rural and urban regions. Annals of Agricultural and Environmental Medicine, 19(3), 529-533.

Pereira, C. A. (2009). Tratado de saúde coletiva em odontologia. Nova Odessa: Napoleão ed.

Rocha MMF. (2014). Avaliação da saúde bucal de crianças e adolescentes submetidos à quimioterapia [Dissertação de mestrado]. Universidade Federal do Piauí, Teresina.

Rodrigues, V. P., Lopes, F. F., Abreu, T. Q., Neves, M. I. R., \& Cardoso, N. C. (2011). Avaliação dos hábitos de higiene bucal de crianças durante o período de internação hospitalar. Revista Odontologia Clínico-Cientifica, 10(1), 49-5.

Silness, J., \& Löe, H. (1964). Periodontal disease in pregnancy. II. Correlation between oral hygiene and periodontal condition. Acta Odontologica Scandinavica, 22(1), 121-126.

Sonis ST; Fazio RC, Fang L. Princípios e prática de medicina oral. Rio de Janeiro: Guanabara Koogan; 1996.

Ximenes, R. C. C., Aragão, D. S. F., \& Colares, V. (2008). Avaliação dos cuidados com a saúde oral de crianças hospitalizadas. Revista da Faculdade de Odontologia, 49(1), 21-25. 